\section{Estilos de aprendizaje y rendimiento académico en estudiantes ingresantes de Odontología}

\author{
Learning styles and their relationship to academic achievement in \\ freshmen students of dentistry
}

\begin{abstract}
Resumen
El presente estudio tuvo como propósito identificar los estilos de aprendizaje y su relación con el rendimiento académico en los estudiantes ingresantes. Metodología: Se utilizó el cuestionario Honey-Alonso de Estilos de aprendizaje (CHAEA), así como el historial académico como evidencia del su rendimiento académico de 48 estudiantes voluntarios, quienes participaron en el programa de Tutoría; se capacitó a docentes, tutores y estudiantes. Resultados: El 32\% de los estudiantes proviene de instituciones educativas públicas y el $78 \%$ de privadas; la edad promedio es de 19 años $\mathrm{DE}+-2,73$. Se encontró que prevalece el estilo teórico $(35,4 \%)$, seguido del activo $(25 \%)$, con una preferencia moderada. Se compararon los estilos de aprendizaje a su ingreso y al término del año académico y se encontraron diferencias en el activo, teórico y pragmático; asimismo se halló relación entre el estilo de aprendizaje reflexivo, teórico y pragmático con el rendimiento académico de los estudiantes. El estilo de aprendizaje reflexivo ha estado muy por debajo del promedio, es necesario impulsarlo pues permite fortalecer la autonomía en el aprendizaje del estudiante, equilibrando el estilo teórico como el activo. Además, al estar inmerso en una institución que brinda servicios de salud, en contacto directo con otro ser humano, se requiere ser cauto, discreto y concienzudo, así como saber observar y escuchar para poder brindar una mejor atención a los pacientes.
\end{abstract}

Palabras clave: Enseñanza aprendizaje, estilos de aprendizaje, rendimiento académico.

\begin{abstract}
This study aimed to identify learning styles preference and their relationship to academic achievement in freshman students. Methodology: Honey-Alonso Learning Styles (CHAEA) questionnaire was used; and evaluation report academic of the freshman students as evidence of their performance 48 student volunteers who participated in the mentoring program; mentor teachers and students were trained. $\mathbf{R e}$ sults: The $32 \%$ of freshman students come from public educational institutions and from $78 \%$ private; the average age is 19 years old and SD +-2.73 . It was found that the theoretical learning style prevails $(35,4 \%)$, followed by active learning style $(25 \%)$ with a moderate preference. Learning styles preference were compared at the beginning and at the end of the academic year, it was founded differences in active, theoretical and pragmatic learning styles; also it was founded relation between reflexive, theoretical and pragmatic learning styles with academic performance of the freshman students. The reflexive learning style has been very below average, it is necessary to motivate them in order to strengthen autonomy in student learning, balancing theoretical style as the active, in addition to being immersed in an institution that provides health services, contact live with another human being, one must be cautious , discreet and thorough, as well as know how to observe and listen to deliver better care to patients, as have the ability of self-education is the most appropriate health professional.
\end{abstract}

Key words: Teaching and learning; learning styles; academic performance
Artículo Original

\author{
Lita Ortiz-Fernández', Hilda Moromi-Nakata², \\ Margot Gutiérrez-Ilave'1, Melissa Barra- \\ Hinostroza², Lita Cáceres de Barcés ${ }^{1}$, \\ Carmen Quintana del Solar ${ }^{3}$, Sylvia Chein- \\ Villacampa ${ }^{1}$, María Núñez-Lizarraga ${ }^{3}$, \\ Juana Bustos de la Cruz $^{3}$ \\ Cecilia Rodríguez-Vargas ${ }^{1}$, Antonia Castro- \\ Rodríguez ${ }^{1}$, Mónica Guillén-Pariona ${ }^{4}$
}

1. Departamento Académico de Estomatología Preventiva y Social

2. Departamento Académico de Ciencias Básicas

3. Departamento Académico de Estomatología Pediátrica

4. Departamento Académico de Estomatología Médico Quirúrgico

1-4.Facultad de Odontología de la Universidad Nacional Mayor de San Marcos, Perú.

\section{Correspondencia}

CD. Lita Ortiz Fernández

Facultad de Odontología de la Universidad Nacional Mayor de San Marcos, Jr. Amézaga 375, Lima 1, Perú.

Correo electrónico: lortizf@unmsm.edu.pe. limaoff@yahoo.es

Coautores:

Moromi- Nakata: <hmnbio@hotmail.com> Barra-Hinostroza; melisbarra@hotmail.com Cáceres de Barcés: litacaceres@yahoo.es Quintana del Solar: carmenquintanadelsolar@gmail.com Chein-Villacampa: sychevi7720@hotmail.com Castro-Rodríguez: afcr21@hotmail.com Nuñez-Lizarraga: mariael24@gmail.com Bustos de la Cruz: cdjrbustos@yahoo.es Rodríguez-Vargas: cecyrv@gmail.com Guillén-Pariona: s5560m@gmail.com

\section{Introducción}

Las instituciones de educación superior, a nivel nacional e internacional, se encuentran con el desafío de impulsar la formación de sujetos autónomos, capaces de participar y tomar decisiones fundamentadas en la vida profesional y social. El aprendizaje es un proceso holístico de la adaptación, no es solo el resultado de la cognición, sino que implica el funcionamiento integral de la persona con un pensamiento total, el sentir, el percibir, el actuar; abarca la resolución de problemas, toma de decisiones, la creatividad.

La autonomía personal en el aprendizaje se refiere a la propia capacidad de aprender a aprender y exige conocer cómo funcionan los procesos que lo hacen posible, conocer los estilos de aprender, disponer de estrategias de aprendizaje adecuadas para construir conocimientos, saber utilizar técnicas que facilitan esos procesos y tener capacidad para utilizarlas en contextos concretos, de acuerdo con las demandas de la tarea que se pretende resolver, dentro de una planificación previamente diseñada para lograr el objetivo de aprendizaje fijado. Por tanto, la autonomía en el aprendizaje requiere el desarrollo 
de un alto nivel de toma de conciencia sobre los propios procesos de aprendizaje, disponer de habilidades metacognitivas sobre los mecanismos cognitivos y su regulación y tener conocimientos significativos sobre aspectos específicos de contenidos ya estudiados, que permitirán la construcción de nuevos significados ${ }^{1}$.

El profesor y los estudiantes tienen funciones bien precisas: el estudiante debe ser responsable de su proceso, de aprendizaje y el docente tiene una intervención mediadora en el proceso; orientado a potenciar el aprendizaje autónomo de los estudiantes, de modo que cada uno sea capaz de elaborar su modelo personal de funcionamiento y acción, con capacidad para regularlo y mejorarlo de manera progresivamente autónoma ${ }^{1}$.

El estudiante de la carrera de Odontología demanda, desde su ingreso, un acompañamiento académico, personal y social, debido a que tiene que superar el difícil tránsito de la educación básica a la universitaria. Si bien es cierto hay déficit en los aprendizajes previos, que pueden ser atribuidos a la formación escolar, no significa que el estudiante carezca de potencial académico, sino que se le hace difícil el proceso de enseñanza aprendizaje; por lo que es necesario proporcionarle destrezas, estrategias y métodos para un mejor aprendizaje. La Tutoría es un espacio que permite una orientación sostenida tanto académica como personal y social, para que los estudiantes sean futuros profesionales competentes, con valores humanos, y un excelente rendimiento académico. Es necesario prevenir y evitar los altos índices de deserción y repitencia de los estudiantes en los primeros ańos de estudios; es todo un reto a enfrentar teniendo en cuenta el número y la heterogeneidad de los estudiantes.

Impulsar el aprendizaje implica brindarles distintas posibilidades de interacción con el conocimiento a través de actividades variadas, que atiendan las diferencias individuales en relación con sus formas y estilos de aprender para un mejor rendimiento académico. La atención de los estudiantes en sus particularidades requiere que el docente conozca cuáles son estos estilos; las condiciones educativas bajo las que un estudiante está en la mejor situación para aprender, o qué estructura necesita para hacerlo mejor; un estilo de aprender no es mejor que el otro, sino son complementarios. El éxito académico del estudiante requiere de un alto grado de compenetración con la misión, visión y fines de la institución educativa.

En la actualidad se ha demostrado que no todos aprendemos de la misma manera, ni al mismo ritmo, porque cada persona utiliza su propio método y estrategia. Los estilos de aprendizaje se definen cómo los rasgos cognitivos, afectivos y fisiológicos que sirven como indicadores estables de como los estudiantes interactuan y responden a sus ambientes de aprendizaje ${ }^{2}$.

El conocimiento del estilo de aprendizaje predominante en las aulas universitarias es una herramienta útil para adaptar el estilo de enseńanza del docente para un mejor rendimiento, a la vez que permite un mejor monitoreo y evaluación del aprendizaje. Asimismo, es de mucha utilidad para el estudiante porque puede planificar su aprendizaje según sus estilos, evitando bloqueos y optimizando resultados ${ }^{2}$.

La auténtica igualdad de oportunidades educativas para los estudiantes no significa que tengan el mismo libro, el mismo horario, las mismas actividades, los mismos exámenes. El estilo de enseñar preferido por el profesor puede significar un favoritismo inconsciente para los alumnos con el mismo estilo de aprendizaje, los mismos sistemas de pensamiento y cualidades mentales.

Es necesario conocer sobre los estilos de aprendizaje y cuál de estos define la forma predilecta de aprender; es importante no solo para los que se supone que aprenden, sino también para los que han asumido la función de enseñar, pues ambos se encuentran relacionados.

\section{Descripción de los estilos de aprendizaje según Honey - Alonso ${ }^{3}$}

- Activos: Gustan de nuevas experiencias. Son de mente abierta, no escépticos y les agrada emprender nuevas tareas. Son personas que viven en el aquí y el ahora.
- Reflexivos: Gustan observar las experiencias desde diferentes perspectivas. Reúnen datos para analizarlos con detenimiento antes de llegar a alguna conclusión. Prefieren ser prudentes y mirar bien antes de actuar.

- Teóricos: Suelen ser perfeccionistas. Por lo general, buscan integrar los hechos en teorías coherentes. Gustan de analizar y sintetizar. Para ellos, la racionalidad y la objetividad son aspectos prioritarios.

- Pragmáticos: Su principal característica se relaciona con la aplicación práctica de las ideas. Son realistas cuando se trata de tomar una decisión o resolver un problema. Su filosofía es: si funciona, es bueno.

Estos estilos incluyen rasgos cognitivos que determinan las formas de conocer de los sujetos; también incluyen los rasgos afectivos: la motivación y las expectativas influyen notablemente en el aprendizaje; los rasgos fisiologicos van desde estudios sobre biotipos y biorritmos, hasta teorías neurofisiológicas, que influyen en el aprendizaje; el proceso perceptivo: influye de modo muy destacado en los procesos de comunicación y aprendizaje; el ambiente de aprendizaje: los individuos perciben, interaccionan y responden a los ambientes de aprendizaje de forma distinta. Los tres rasgos serían relativamente estables, es, decir, pueden cambiar con esfuerzo y tecnicas adecuadas ${ }^{3,4}$.

Definir rendimiento académico es muy complejo porque tiene una variedad de interpretaciones. En la educación superior, el rendimiento académico ha sido clasificado de varias maneras: Se distingue el rendimiento parcial del general. El primero corresponde a los requerimientos parciales alcanzados por los estudiantes en cada una de sus actividades académicas; es decir, la aprobación de sus asignaturas correspondientes en la secuencia temporal prevista por su plan de estudios, así como el nivel de logro cognitivo alcanzado de cada materia aprobada. En cambio, la idea de concepto general sería ponderar los éxitos y fracasos del alumno durante su trayectoria, para lo cual se debería relacionar el éxito con el nivel de conocimientos aprendidos.

Descripción de los estilos de aprendizaje según Honey - Alonso

\begin{tabular}{llll}
\hline Estilos & $\begin{array}{l}\text { Contenidos declarativos } \\
\text { y conceptuales }\end{array}$ & $\begin{array}{l}\text { Contenidos } \\
\text { procedimentales }\end{array}$ & $\begin{array}{l}\text { Contenidos } \\
\text { estratégicos }\end{array}$ \\
\hline Activo & Poco interés & Mucho interés & Relativo interés \\
Reflexivo & Relativo interés & Poco interés & Mucho interés \\
Teorico & Mucho interés & Poco interés & Relativo interés \\
Pragmático & Poco interés & Relativo interés & Mucho interés \\
\hline
\end{tabular}


Tejedor ${ }^{5}$ considera tres modos de aproximación al rendimiento académico inmediato en la educación superior. El primero plantea el rendimiento en un sentido amplio, a través de diferentes resultados: el éxito (culminación puntual de una carrera en los años previstos en el plan de estudios), el retraso (finalización empleando más tiempo del establecido oficialmente) y el abandono de los estudios. El segundo es la regularidad académica, la cual se mide por medio de las tasas de presentación o no a los exámenes; mientras que el último modo de aproximación sería el rendimiento en sentido estricto: las notas o calificaciones alcanzadas. A pesar del riesgo que implica usar exclusivamente las calificaciones para medir el rendimiento académico, debido fundamentalmente a la subjetividad de los docentes, las calificaciones no dejan de ser el medio más usado para operacionalizar el rendimiento académico ${ }^{5}$.

Desde un punto de vista práctico, lo habitual es identificar rendimiento con resultados, debiendo distinguirse entre estos dos categorías: inmediatos y diferidos. Los primeros estarían determinados por las calificaciones que obtienen los alumnos durante su carrera hasta la obtención del título correspondiente y se definen en términos de éxito/fracaso en relación con un determinado período temporal. Por otro lado, el rendimiento diferido hace referencia a su conexión con el mundo del trabajo, en términos de eficacia y productividad; se vincula, sobre todo, con criterios de calidad de la institución.

Referirnos al rendimiento inmediato, también precisa y hace diferencia a su vez entre dos tipos de rendimiento por una parte, el rendimiento en sentido estricto, medido a través de la presentación a exámenes o éxito en las pruebas (calificaciones), que se traduce en unas determinadas tasas de promoción (superación de curso), repetición (permanencia en el mismo curso más de un año) y abandono (alumnos que dejan de matricularse en cualquiera de los cursos de la carrera); por otra, el rendimiento en sentido amplio, medido a través del éxito (finalización puntual en un período de tiempo determinado) o del fracaso (retraso o abandono de los estudios). También se habla de regularidad académica, cuando el concepto de rendimiento académico se presenta mediante las tasas de presentación o no a las convocatorias de exámenes. Igualmente, en este concepto de rendimiento en sentido amplio, cabe incluir la referencia al tratamiento de las actitudes de los alumnos hacia los estudios, hacia la institución. La consideración, por otra parte, de las condiciones institucionales que pueden contribuir a producir un determinado rendimiento en el alumno nos conduce directamente a la necesidad de valorar la satisfacción de los alumnos con dichas condiciones. Queda así concretada nuestra concepción amplia del rendimiento: calificaciones, tasas de éxito/fracaso (individuales y colectivas), actitudes y satisfacción ${ }^{5}$.

Este estudio asume la definición:"el rendimiento académico apropiado puede hacer referencia a aspectos muy diversos, entre los cuales destacan: terminar un semestre con todas las asignaturas aprobadas, no repetir semestres o asignaturas, no abandonar la carrera, realizar todos los exámenes y aprobarlos". Estas condiciones no se observan en los estudiantes de bajo rendimiento académico. Por estas razones, el uso de estilos de aprendizaje juega un papel determinante en la formación académica de los estudiantes de nivel superior $^{5,6,7}$.

La Ley Universitaria Peruana No 23733 (1983-2010), en su artículo 57, dice en relación con el rendimiento académico: "Son deberes de los estudiantes: a) dedicarse con esfuerzo y responsabilidad a su formación humana, académica y profesional. c) Contribuir al prestigio de la Universidad y a la realización de sus fines. d) Aprobar las materias correspondientes al período lectivo, caso contrario perderán la gratuidad de la enseñanza"8.

En una universidad pública de México, se caracterizó e identificó la relación de estilos de aprendizaje y el rendimiento académico de estudiantes. El estilo predominante fue el reflexivo, seguido por el pragmático, el teórico y el activo, con una preferencia moderada en cada estilo; en lo relativo al rendimiento académico, el promedio general de los participantes fue de 86.83 puntos de 100 , siendo el promedio mínimo de 65 y el máximo de 98 . Se encontraron diferencias significativas entre estilos de aprendizaje y rendimiento académico ${ }^{9}$. En la identificación del perfil de los estilos de aprendizaje en estudiantes de primer año en la Universidad de Chile, se encontró que predominaba el estilo pragmático 9 .

La evaluación de los estilos de aprendizaje puede contribuir a una aproximación más personalizada de la consejería estudiantil, la estrategia instruccional y la evaluación del aprendizaje.
La investigación se justifica desde los ámbitos académico y organizativo: la educación superior cumple un papel esencial en la promoción de la cohesión social, con la reducción de las desigualdades y en la elevación del nivel del conocimiento, destrezas y competencias en el seno de la sociedad; se requiere unir esfuerzos para facilitar servicios adecuados a los estudiantes, crear itinerarios de aprendizaje más flexibles a fin de acceder a la educación superior y ampliar la participación a todos los niveles sobre la base de igualdad de oportunidades. El conocimiento del estilo de aprendizaje predominante en las aulas universitarias es una herramienta útil para adaptar el estilo de enseñanza del docente para un mejor rendimiento del estudiante, a la vez que permite el monitoreo y evaluación del aprendizaje. Asimismo, es de utilidad para el estudiante porque le permite planificar su aprendizaje según sus estilos, evitando los bloqueos y optimizando resultados.

Desde el punto de vista académico, los docentes tutores están comprometidos en mejorar el proceso de enseñanza aprendizaje permitiendo un abordaje inclusivo, lo que redundará en realizar intervenciones de calidad. Identificar los estilos de aprendizaje que tienen los estudiantes es necesario para que se fortalezcan y/o se adapten metodologías de enseńanza desde los docentes, formentando el aprendizaje para elevar la calidad educativa ${ }^{2,4,5}$.

Desde el ámbito organizativo, son elementos vitales para propiciar espacios de aprendizaje y el intercambio de experiencias entre los diferentes departamentos académicos, instituciones y países que han realizado estudios de este tipo.

Las nuevas tendencias en educación superior confieren más atención a los procesos de aprendizaje de los estudiantes como respuesta a la demanda social de formar personas capaces y eficaces. Considerando estas tendencias, el aprendizaje se caracteriza por ser un proceso dinámico, continuo, global, personal y gradual que surge de la experiencia individual ${ }^{1}$. Por ello, las teorías de estilos de aprendizaje se han convertido en una alternativa para explicar el motivo por el cual, en un mismo grupo, los estudiantes aprenden de manera diferente $e^{4}$.

Esta investigación tiene como objetivo identificar los estilos de aprendizaje en los estudiantes ingresantes al inicio y al final de cursar el primer ańo en la 
Facultad de Odontología y su relación con el rendimiento académico.

\section{Materiales y método}

Este estudio es cuasiexperimental, cuanticualitativo, comparativo. La población de estudio son los estudiantes ingresantes de la Facultad de Odontología de la Universidad Nacional Mayor de San Marcos. Las unidades de observación se constituyen 48 estudiantes que han participado en el Programa de Tutoría en forma voluntaria. Las unidades de información son 8 docentes tutores.

Criterios de inclusión: estudiantes matriculados con asistencia regular, con características similares en cultura y estrato social. Se solicitó consentimiento informado a los estudiantes y docentes que participaron.

El estudio tuvo tres fases. En la primera, se identificaron los estilos de aprendizaje iniciales de los estudiantes ingresantes y su perfil sociodemográfico, luego se realizó una capacitación a los docentes tutores sobre las formas de aprender y otra sobre los estilos de aprendizaje. En la segunda, se conformaron los grupos de trabajo tutorial, monitoreados por un docente tutor, quienes capacitaron a los estudiantes sobre estilos de aprendizaje y rendimiento académico. En la tercera se evaluaron los estilos de aprendizaje después de la intervención y la relación con su rendimiento académico en el primer año.

Al estudiante se le orientó acerca de cómo controlar su propio aprendizaje, identificar sus fortalezas y debilidades, describir sus estilos de aprender cómo aprender, día a día y cómo superar las dificultades.

Fase I.- Los estilos de aprendizaje se midieron a través del cuestionario de estilos de aprendizaje CHAEA (Honey y Alonso, 1997), el cual es una contribución de la Dra. Catalina Alonso (1992), quien recogió las experiencias de Honey y Mumford (1986) y adaptó el cuestionario LSQ de estilos de aprendizaje al ámbito académico y al idioma español y lo dirigió a los estudiantes universitarios, añadiendo una serie de preguntas socioacadémicas y llamó al cuestionario adaptado (CHAEA) como cuestionario de Honey - Alonso de estilos de aprendizaje. Es un instrumento ya validado en muchas poblaciones de habla hispana; tiene muy buenos resultados, por lo que se decidió aplicarlo por primera vez en nuestra institución. Este cuestionario identifica cuatro estilos de aprendizaje: activo, reflexivo, teórico y prag- mático, y consta de 80 preguntas que contestan + (más) significa de acuerdo total y - (menos) en total desacuerdo, se encuentran divididas en cuatro secciones de veinte preguntas cada una, las que se refieren a los cuatro estilos de aprendizaje. El nivel de confiabilidad es considerado como aceptable y cuenta con validez de constructo $^{10}$. Se utilizó una escala preestablecida para determinar preferencia con los valores por cada estilo de aprendizaje de muy alto, alto, moderado, bajo y muy bajo, como base para una propuesta pedagógica ${ }^{11}$.

Para el rendimiento académico se asumió la definición de terminar un semestre con todas las asignaturas aprobadas 5 , no repetir asignaturas, no abandonar la carrera; se les categorizó como: (sí) aprobó las siete asignaturas o (no) aprobó alguna de las siete asignaturas, complementándolo con sus discursos más representativos. Para la aplicación del instrumento, se solicitó autorización al director de la escuela académico profesional para realizar la actividad en los espacios del Programa de Tutoría. Se identificó la referencia de estilos de aprendizaje de los estudiantes ingresantes y su perfil sociodemográfico. Se obtuvieron los discursos más representativos sobre los significados de rendimiento académico y estilos de aprendizaje. Luego se capacitó en dos oportunidades a los estudiantes sobre estilos de aprendizaje.

Fase II.- Se conformaron 8 grupos con 5 estudiantes participantes de forma voluntaria, liderados por un docente tutor $^{12,13,14,}$ quien monitoreó el desempeño académico y acompañó al estudiante durante cuatro fechas en forma individual.

Se capacitó a los docentes tutores en cuanto a los estilos de aprendizaje y la caracterización de sus tutorados; también se instruyó a los estudiantes ingresantes sobre la importancia de involucrar más estilos de aprendizaje pues estos influyen en un mejor desempeño. Los encuentros con los estudiantes y tutores tuvieron dificultades, por no tener horarios comunes, por lo que un $25 \%$ de los estudiantes tuvieron cuatro sesiones, mientras que el $20 \%$ tuvo tres y el $55 \%$ tuvo solo dos encuentros. Esto fue una limitante para un mejor acompañamiento del estudiante.

Fase III.- Se identificó la preferencia de los estilos de aprendizaje de los estudiantes ingresantes después de la intervención tutorial; mientras que el rendimiento académico se obtuvo del promedio de las calificaciones de los participantes. Se recopilaron los discursos más representativos en relación con el porqué de su rendimiento académico para complementar los aspectos actitudinales y no centrarse solo en el aspecto cognitivo.

Se comparó el rendimiento académico con las calificaciones obtenidas y los estilos de aprendizaje antes y después de la intervención con medidas de tendencia central y prueba de Wilcoxom.

\section{Resultados}

Se caracterizó el perfil del estudiante ingresante, siendo la edad promedio de 19,41 ańos con una $\mathrm{DE}+/$ - de 2,73; según género, el $46 \%$ es femenino y el 54 $\%$ masculino; en cuanto a la institución Educativa de procedencia, el $32 \%$ proviene de estatal y el $68 \%$ de particular.

Se identifica la preferencia de los estudiantes según estilo de aprendizaje inicial, predominando el teórico con un $72.9 \%$, seguido del activo con un 52\%.

El estilo teórico tiene el criterio de muy alto y alto el $14.6 \%$, moderado el $35,40 \%$, mientras que el $50 \%$ es bajo y muy bajo; al final el $33.3 \%$ es muy alto y alto, moderado un $29,20 \%$, bajo y muy bajo un $37.5 \%$ (Gráf. N²1).

En cuanto al estilo de aprendizaje activo, se encontró al inicio con el criterio de muy alto y alto en un 4,20 \% del total, siendo moderado el $25 \%$, mientras que el $70.8 \%$ es bajo y muy bajo; al final se halló el estilo activo como muy alto y alto en un $25 \%$, siendo moderado un $39,60 \%$, el bajo y muy bajo fue el 35.5\%. (Gráf. №2).

El estilo reflexivo no está presente como muy alto, mientras que un $6,30 \%$ lo tiene alto, siendo moderado el $22,90 \%$, el $70.90 \%$ es bajo y muy bajo; al final el reflexivo se mantuvo sin tener el criterio de muy alto, siendo alto el $8,30 \%$, moderado un $31,30 \%$, el $60.4 \%$ como bajo y muy bajo. (Gráf. N³).

Correspondiendo al pragmático de muy alto y alto el $6.3 \%$, moderado el 16,70 $\%$, mientras que el $76.2 \%$ es bajo y muy bajo; siendo al final el $14.60 \%$ como muy alto y alto, moderado un $29,10 \%$, mientras que el $56.3 \%$ es bajo y muy bajo (Gráf. № 4 ).

En cuanto al rendimiento académico se identificó que el $81 \%$ de los estudiantes participantes aprobaron todas las asignaturas del segundo semestre, mientras que el $19 \%$ desaprobó una o más. Los valores difieren al del año 2011, en el que el $80 \%$ estuvo aprobado y el $20 \%$ desaprobó. 
Se halló que los estilos de aprendizaje activo, teórico y pragmático son diferentes al inicio de la etapa universitaria y al término del primer año de estudios (Prueba de Wilcoxom) (Tabla 1).
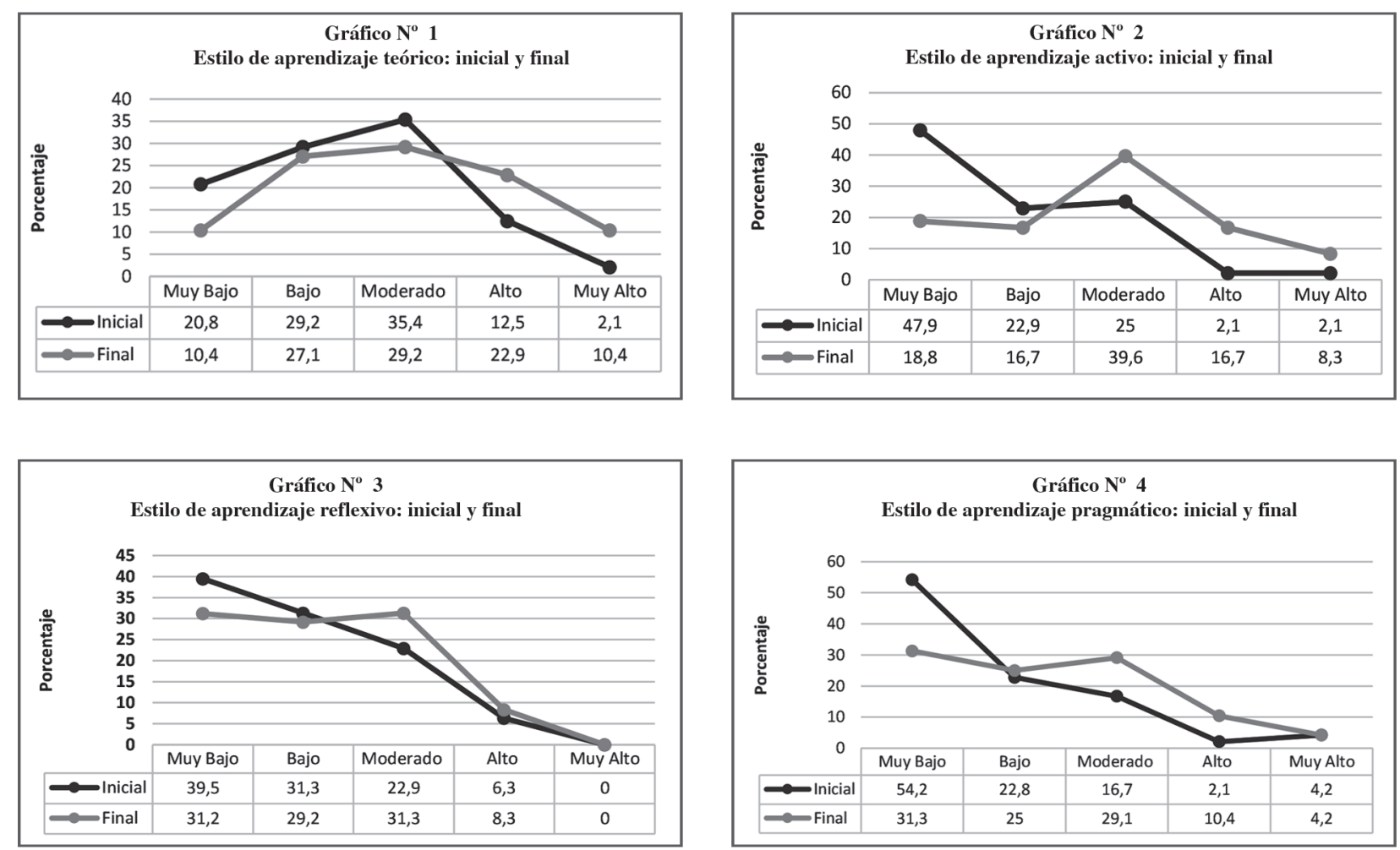

Tabla 1. Estilos de aprendizaje al ingreso y al término del primer año

\begin{tabular}{ll}
\hline $\begin{array}{l}\text { Estilos de } \\
\text { aprendizaje }\end{array}$ & $\begin{array}{l}\text { Nivel de } \\
\text { significancia }\end{array}$ \\
\hline Activo & $0.000^{*}$ \\
Reflexivo & 0.155 \\
Teórico & $0.009^{*}$ \\
Pragmático & $0.033^{*}$ \\
\hline$\left(\mathrm{Z}={ }^{*} \mathrm{p}<0.05\right)$ Wilcoxon &
\end{tabular}

Tabla 2. Estilos de aprendizaje final según rendimiento académico

\begin{tabular}{ll}
\hline $\begin{array}{l}\text { Estilos de } \\
\text { aprendizaje }\end{array}$ & $\begin{array}{l}\text { Nivel de } \\
\text { significancia }\end{array}$ \\
\hline Activo & 0.069 \\
Reflexivo & $0.006^{*}$ \\
Teórico & $0.000^{*}$ \\
Pragmático & $0.019^{*}$ \\
\hline$\left(\mathrm{Z}={ }^{*} \mathrm{p}<0.05\right)$ &
\end{tabular}

Se halló que los estilos de aprendizaje reflexivo, teórico y pragmático finales están relacionados con el rendimiento académico (Prueba Kruskal Wallis) (Tabla 2).

El cuestionario de Honey Alonso permitió la descripción de los estilos de aprendizaje característicos en los estudiantes ingresantes y su relación con el rendimiento académico.

En cuanto a los discursos más representativos sobre el aprendizaje y estilos de aprender, tomamos en cuenta lo que nos expresaron 5 estudiantes, 2 mujeres, y 3 varones.

M1 (F) "Se me hace complejo aprender solo mirando o escuchando, tengo mayor facilidad de entender cuando estoy haciendo algo y me orientan”.

M2 (F) "Tengo dificultades en poder hacer las prácticas, siento que todo es muy rápido, no tengo tiempo de consolidar mis conocimientos, tengo dudas, tengo que asistir en otro horario para evitar jalarme”.

M3 (M) “ Me interesa aprender y también tener buenas notas, pero eso está condicionado también a que tengamos diversas formas de que nos enseñen para poder aprender mejor, predomina más la clase magistral y he tenido que acostumbrarme en degrabar las clases, porque de ahí viene todo".

J5 (M) "Me gustaría tener tiempo para entender sobre las diversas formas de aprender, pero mi tiempo es tan corto que no puedo asistir a las otras citas pendientes con mi tutora”.

F7 (M) "Predominan las clases magistrales, creo que deben hacer otro tipo de propuestas, hay veces que nos cansamos con este tipo de propuesta”.

\section{Discusión}

Cuando el estudiante inicia sus estudios en la Universidad, se espera que sea competente con capacidad de autorregularse; es necesario tener en cuenta que la autorregulación del aprendizaje puede enseñarse, que no se adquiere de una sola vez y para siempre. El aprendizaje se concibe mejor como un proceso, no en términos de resultados. Para mejorar el aprendizaje en la educación superior, el objetivo principal debe ser la participación activa de los estudiantes en el proceso que fortalezca su aprendizaje, que incluya la eficacia de sus esfuerzos de aprendizaje.

Los procesos educativos son el resultado de múltiples reformas que en los últimos años han dado un giro hacia la autoformación y el autoaprendizaje; se requiere un esfuerzo sostenido del docente de educación básica hasta el de la Universidad para formarse y asumir estilos y formas de enseńar que permitan dar respuesta a las necesidades de autoaprendizaje de los alumnos; más aún si son de una carrera de salud, porque al estar inmersos en una institución que brinda servicios de salud, en contacto directo con otro ser humano, se requiere ser cauto, discreto y concienzudo, así como saber observar y escuchar para 
poder brindar una mejor atención a los pacientes, como tener la capacidad de autoformación y esto se caracteriza en el estilo reflexivo de aprender. Este es, sin duda, el más apropiado que debe contar un profesional de salud.

Díaz A. en Perú, el 2012, identificó en la Universidad Técnica del Callao, que los estudiantes de primer año a su ingreso tienen predominio del estilo teórico $(51 \%)$, seguido del activo $(21 \%)$; mientras que en esta investigación se encontró que prevalece el teórico $(35,4 \%)$, seguido del activo (25\%), con una preferencia moderada. Asimismo halló relación entre el estilo de aprendizaje con el rendimiento académico de los estudiantes ingresantes; coincidiendo con el presente estudio en el teórico, mas no en el reflexivo ${ }^{15,16}$.

Juárez Lugo C. y colaboradores en México 2011-UAEM identificaron, que en los estudiantes de primer semestre predomina el estilo reflexivo (13.84\%), seguido del pragmático (12.68); mientras que el presente estudio encontró que prevalece el teórico (35.4\%), seguido del activo (25\%), con una preferencia moderada. Asimismo, no halló relación entre los estilos de aprendizaje con el rendimiento académico de los estudiantes ingresantes; mientras que el presente estudio sí, tanto en el teórico como en el reflexivo ${ }^{2}$.

Napoli J. y colaboradores en Argentina, el 2010, realizaron una evaluación comparativa de los estilos de aprendizaje en estudiantes de pregrado de Patología, médicos residentes y profesionales de la carrera docente y encontraron que predominaba el estilo reflexivo; no concordando con el presente estudio.

\section{Conclusiones}

El estilo de aprendizaje que predomina en el estudiante ingresante al iniciar sus estudios universitarios es el teórico, si bien es cierto se caracteriza porque adapta e integra las observaciones y los fundamentos de las teorías complejas, sintetiza la información y actúa con lógica y racionalidad; también representa que aún está en proceso de construcción de la autonomía y requiere de una estructura de aprendizaje, de un método y un orden.

El estilo de aprendizaje reflexivo ha estado muy por debajo del promedio, es necesario impulsarlo pues permite for- talecer la autonomía en el aprendizaje del estudiante, equilibrando el estilo teórico y activo, con capacidad analítica, prudenciva y juicio.

Los estilos de aprendizaje al inicio y al final del primer año tienen diferencias en los estilos de aprendizaje activo, teórico y pragmático.

Los estilos de aprendizaje reflexivo, teórico y pragmático finales están relacionados con el rendimiento académico de los estudiantes ingresantes.

El acompañamiento individualizado permite dar atención a la diversidad de los estudiantes según sus estilos de aprendizaje.

Los resultados pueden ser herramientas facilitadoras para la retroalimentación y operacionalización al modelo pedagógico institucional.

\section{Recomendaciones}

Es necesario impulsar el desarrollo del estilo de aprendizaje reflexivo, pues permitirá fortalecer la autonomía en el aprendizaje del estudiante y su capacidad analítica.

Es ineludible acercar al estudiante a experiencias de aprendizaje que movilicen el desarrollo de todas sus potencialidades, siendo el docente el gestor de ellas.

Es necesario continuar esta línea de investigación en todos los años de estudios, para fortalecer capacidades en los estudiantes y docentes.

Es ineludible continuar las investigaciones relacionando estilos de aprendizaje con estilos de enseñanza.

Establecer una evaluación permanente de los estilos de aprendizaje y rendimiento académico, involucrando satisfacción, bienestar y éxito académico.

\section{Agradecimiento}

Al Vicerrectorado de Investigación de la Universidad Nacional Mayor de San Marcos, por el apoyo financiero, operativo, de consultoría y administrativo que permitió desarrollar el presente estudio.

Asimismo, a las docentes tutoras que se comprometieron en orientar y hacer consejería a los estudiantes ingresantes.

Esta investigación se expuso en el VI Congreso Mundial de Estilos de Aprendizaje, realizado en la Pontificia Uni- versidad Católica del Perú en mayo del 2014; en la mesa temática Estilos de aprendizaje y estilos de enseñanza, nuevos enfoques, ámbitos y formas de intervención.

\section{Referencias bibliográficas}

1. Caballero SC. ¿Qué aprendizaje promueve el desarrollo de competencias? Una mirada desde el aprendizaje significativo. Revista Qurriculum 2009:11-34

2. Juárez CS, Hernández-Castro SG, Escoto MC. Rendimiento Académico y estilos de aprendizaje en estudiantes de Psicología. Revista Estilos de Aprendizaje 2011; 7 (7): 79-92.

3. Laugero L, Balcaza G, Salinas N, Craveri AM. Una indagación en el estilo de aprendizaje de los alumnos en distintos momentos de su vida universitaria. Revista Estilos de Aprendizaje 2009; 4(4):1-13.

4. Salim R. Motivaciones, enfoques y estrategias de aprendizaje en estudiantes de Bioquímica de una universidad pública argentina. Revista Electrónica de Investigación Educativa [Internet]. 2006 [citado 2011-12-2]; 8(1). Disponible en: http://redie.uabc.mx/vol8no1/ contenido-salim.html

5. Tejedor FJ y Muñoz-Repiso AGV. Causas del bajo rendimiento del estudiante universitario (en opinión de los profesores y alumnos): propuestas de mejora en el marco del EEES. Revista de Educación 2007; (342): 419-442.

6. Morales DG, Alfonso YMD. (2006). La importancia de promover en el aula estrategias de aprendizaje para elevar el nivel académico en los estudiantes de Psicología. Revista Iberoamericana de Educación [Internet]. 2006 [citado 2012-12-02]; 40(1):8. Disponible en: http://www.reoie.org/ investigacion/137gonzalez.pdf

7. Pérez MV, Díaz-Mujica A, González-Pienda JA, Núñez JC. Docencia para facilitar el aprendizaje activo y autorregulado. Revista Diálogo Educacional 2010; 10(30): 409-424.

8. Ley Universitaria No 23733. 1983-2010 [citado 2011-12-2]. 
Disponible en: http://www.peru. gob.pe/docs/PLANES/10402/ PLAN_10402_Ley\%20Universitaria_2010.pdf.

9. Pierart CGA, Valdebenito VM, Salgado MTC, Palma HM, Seiffert KR. Perfil de estilos de aprendizaje en estudiantes de primer ańo de dos carreras de diferentes áreas de la Universidad en la Concepción. Revista de estilos de aprendizaje 2009; 3(3):56-69.

10. Alonso C, Gallego D, Honey P. Los Estilos de Aprendizaje: Procedimientos de diagnóstico y mejora. 6a ed. Bilbao: Ediciones Mensajero; 1994.

11. Cue J. Los estilos de Aprendizaje como base a una propuesta pedagógica. 2010 [citado 2010-08-20]. Disponible en: http ://www.slideshare.net/jlgcue/estilos-de-aprendizaje-presentation-957731
12. Honey P, Mumford A. "The Manual of Learning Styles". 3a ed. Reino Unido: Maidenhead, Berkshire. 1992.

13. Barros DMV, dos Reis VL. A função tutorial na formaçáo continuada docente (the mentoring function in continuing education). Ried. Revista iberoamericana de educación a distancia 2009; 12(1):37-62.

14. Mansilla PU y Escribano ML. Estudio contrastivo de los estilos de aprendizaje en los estudiantes de arquitectura. Didáctica Lengua y Literatura. 2002; 14(2): 251-271.

15. Díaz A. Relación entre los estilos de aprendizaje y el Rendimiento académico de las estudiantes de la Escuela profesional de enfermería de la Facultad de Ciencias de la Salud de la Universidad Nacional del Callao Informe de Investiga- ción. UTC. Callao. Perú; 2012. [citado 2012-12-2]. Disponible en: http://www.unac.edu.pe/ documentos/organizacion/vri/ cdcitra/Informes_Finales_Investigacion/IF_NOVIEMBRE_2012/ IF_DIAZ\%20TINOCO_FCS/ INFORME\%20DE\%20INVESTIGACION.pdf

16. Ortiz-Fernández L et al. Estrategias, estilos de aprendizaje y rendimiento académico en estudiantes ingresantes de Odontología. Odontol Sanmarquina, 2015; 17 (2): 76-81.

17. Napoli J, Formosa MI, Urssi L. Evaluación comparativa de los estilos de aprendizaje en estudiantes de pregrado de Patología, médicos residentes y profesionales de la $\mathrm{Ca}$ rrera docente. Rev Assoc Med Arg. 2010; 123(4):18-22. 